\title{
Observations of sound-speed fluctuations on the New Jersey continental shelf in the summer of 2006
}

\author{
John A. Colosi \\ Department of Oceanography, Naval Postgraduate School Monterey, California 93943 \\ Timothy F. Duda, Ying-Tsong Lin, James F. Lynch, and Arthur E. Newhall \\ Woods Hole Oceanographic Institution, Woods Hole, Massachusetts 02543 \\ Bruce D. Cornuelle \\ Scripps Institution of Oceanography, La Jolla, California 92093
}

(Received 22 December 2010; revised 22 September 2011; accepted 1 October 2011)

\begin{abstract}
Environmental sensors moored on the New Jersey continental shelf tracked constant density surfaces (isopycnals) for 35 days in the summer of 2006. Sound-speed fluctuations from internal-wave vertical isopycnal displacements and from temperature/salinity variability along isopycnals (spiciness) are analyzed using frequency spectra and vertical covariance functions. Three varieties of internal waves are studied: Diffuse broadband internal waves (akin to waves fitting the deep water Garrett/Munk spectrum), internal tides, and, to a lesser extent, nonlinear internal waves. These internal-wave contributions are approximately distinct in the frequency domain. It is found that in the main thermocline spicy thermohaline structure dominates the root mean square sound-speed variability, with smaller contributions coming from (in order) nonlinear internal waves, diffuse internal waves, and internal tides. The frequency spectra of internal-wave displacements and of spiciness have similar form, likely due to the advection of variable-spiciness water masses by horizontal internal-wave currents, although there are technical limitations to the observations at high frequency. In the low-frequency, internalwave band the internal-wave spectrum follows frequency to the -1.81 power, whereas the spice spectrum shows a -1.73 power. Mode spectra estimated via covariance methods show that the diffuse internal-wave spectrum has a smaller mode bandwidth than Garrett/Munk and that the internal tide has significant energy in modes one through three. (C) 2012 Acoustical Society of America.
\end{abstract}

[DOI: 10.1121/1.3666014]

PACS number(s): 43.30.Es, 43.30.Ft [DRD]

Pages: $1733-1748$

\section{INTRODUCTION}

Sound transmission in shallow-water environments displays inherent instabilities or fluctuations in both space and time due to environmental effects. Variations in the ocean sound-speed field are one significant contribution to the observed acoustic variability, and have received much attention, especially for acoustic frequencies in the tens to hundreds of hertz range (see Apel et al., 1997; Apel et al., 2007; Lynch et al., 2005, and references therein). Important ocean processes that have been identified to contribute to watercolumn sound-speed field fluctuations are: Shelf eddies and associated filaments, meandering fronts, and internal waves and tides. Shallow-water internal waves and tides have garnered much attention because of their large vertical water parcel displacements, $\xi$, and their relatively short space and time scales. These waves cause sound-speed fluctuations given to first order by

$$
\delta c=\left(\frac{d c}{d z}\right)_{p} \xi,
$$

where $(d c / d z)_{p}$ is the potential sound-speed gradient, and they can impose significant limitations to acoustic remote sensing, communication, and navigation in the shallowwater environment. However, although internal waves vertically move surfaces of constant density (isopycnals), thus altering the sound-speed profile in a simple kinematic process, submesoscale stirring and adjustment processes generate sound-speed profile changes in another way (Young, 1994). These processes produce density compensated temperature $(T)$ and salinity $(S)$ variations along isopycnals (Phillips, 1971; Munk, 1981; Ferarri and Rudnick, 2000). Compensated $T$ and $S$ anomalies have reinforcing effects on sound speed (Dzieciuch et al., 2004). This density compensated $T$ and $S$ structure is termed spicy thermohaline structure (Munk, 1981), and sometimes simply "spice." The advection of this structure by horizontal internal-wave currents can be a primary contaminant in estimating internalwave properties from temperature-only measurements (Garrett and Munk, 1971). Although physical oceanographers have studied spiciness for decades, ocean acousticians have not, in general, been able to quantify the magnitude or the scales of spicy sound-speed anomalies in their experiments, let alone come to an understanding of the impact on the observed acoustic fields [some noteworthy exceptions are Dzieciuch et al. (2004) and the numerical study by Ewart (1980)]. Spicy thermohaline structure is known to be most significant in the upper ocean where vigorous mixing is occurring, where there is an active eddy field, or where there is significant intermingling of water masses (Munk, 1981). For these reasons continental shelf or shallow-water regions can be expected to have strong sound-speed fluctuations due 
to spiciness. This paper provides a unique examination of spicy thermohaline sound-speed structure in a shallow-water acoustic experiment. The structures are contrasted with the kinematic sound-speed fluctuations caused by shallow-water internal waves. Knowledge of shallow-water internal-wave and spice space-time scales are crucial inputs to acoustic fluctuation calculations, and the presentation here is aimed at facilitating this effort [an example of the application of these results is given by Colosi et al. (2012)].

In this study, data from a mooring hosting ten closely spaced, high precision $T$, conductivity $(C)$, and pressure $(P)$ sensors deployed during the Shallow Water 2006 (SW06) experiment on the New Jersey continental shelf are used to track isopycnals and the $\mathrm{T} / \mathrm{S}$ variation along the isopycnals over a 35 day duration. These measurements are unique as previous shallow-water acoustics experiments have exclusively utilized temperature only instrumentation or sparse TCP observations, which irreconcilably co-mingle internalwave and spice sound-speed effects (Apel et al., 1997; Colosi et al., 2001; Duda et al., 2004, to mention a few). Commonly used acoustic Doppler current profilers (ADCPs) provide useful observations of internal-wave horizontal currents, but a dispersion relation is needed to connect the horizontal current information to the acoustically important vertical displacements. Because spicy structure is along density surfaces, there are no associated currents and thus ADCPs do not measure spice. The observations presented in this paper, then, represent the highest quality view of shallow-water sound-speed fluctuations from internal waves and spice that have been obtained to date.

The observations do have some inherent limitations. For example, tracking isopycnals in depth requires interpolation between sensors and thus more instruments on the mooring would help the analysis (particularly in the steep main thermocline). Further, present day technologies have difficulty measuring salinity in rapidly changing environments as the salinity value is inferred from conductivity, which depends mainly on temperature. Thus, the conductivity sensor must respond quickly to changing ocean temperatures in order to provide an accurate salinity (and thus density) observation (Mensah et al., 2007). In this shallow-water environment nonlinear internal waves cause rapid changes that lead to response time limitations of our conductivity measurement at high frequencies.'

For an acoustic propagation experiment, however, the SW06 observations provide an adequate data set to make first-order statements concerning internal-wave and spicy sound-speed statistics. As such, the root mean square (rms) sound-speed variations along several isopycnals from spice are computed as a function of mean isopycnal depth and they are compared to contributions from three different varieties of shallow-water internal waves, namely diffuse broadband internal waves similar to those described by the Garrett-Munk deep-water internal-wave model, semidiurnal internal tides, and nonlinear internal waves such as internal solitary waves. It is found that spicy effects dominate the sound-speed fluctuation field for time scales between minutes and the inertial day, although it must be pointed out that the rms metric is a deceivingly low metric for the strong but episodic nonlinear internal waves. In addition, frequency spectra of internal waves and spiciness are seen to have similar form consistent with the well-established view that internal-wave horizontal currents advect the spicy structure, thus strongly influencing the spectra (Garrett and Munk, 1971; Munk 1981). In the frequency range between twice the Coriolis frequency and $\sim 1$ cycle per hour (cph), the spectra show a power-law behavior with exponent of $-1.81 \pm 0.25$ for internal waves and $-1.73 \pm 0.20$ for spice. An inversion for the diffuse internalwave and internal tide mode spectra using the depth covariance functions show that the diffuse internal-wave spectrum has a Garrett-Munk form $1 /\left(j^{2}+j_{*}^{2}\right)$ with $j_{*}^{2}=3$, and the internal tide has significant energy in modes $1-3$. The present results for the diffuse internal-wave spectra are found to be consistent with those of Apel et al. (1997), but not of Yang and Yoo (1999), both studies of which analyze data from the Mid-Atlantic Bight.

The outline of this paper is as follows. In Sec. II, the SW06 experiment is briefly discussed, and the computation of isopycnals surfaces and their associated depth, $T$ and $S$ variability are presented. Frequency spectra and statistics for internal waves, as well as a spicy thermohaline structure, are presented in Sec. III. Section IV presents an analysis of the vertical structure of these processes and summary and conclusions are given in Sec. V.

\section{THE SW06 EXPERIMENT}

The SW06 experiment was an interdisciplinary and multiinstitutional effort carried out on the New Jersey continental shelf with the primary goal of studying the effects of various environmental processes on shallow-water acoustic propagation. Areas of interest included bio-acoustics, geo-acoustics, and a strong focus on physical oceanography and water column acoustics. Many details of the experiment are summarized by Lynch and Tang (2008), and are not repeated here.

Of relevance to this study was the deployment of a single mooring carrying ten closely spaced Sea-Bird Electronics Inc. (SBE) pumped $T, C$, and $P$ sensors (SBE37 SMP) to enable precise observations of salinity $(S)$ and thus density. The pumping capability, which is new in the last decade and has been the standard on ship board temperature, conductivity, and depth (CTD) applications, helps ensure that temperature (measured external to the conductivity cell) and conductivity (measured in the cell) are measured for a single and identifiable fluid parcel. Salinity is estimated by measuring conductivity of a sample at a known temperature. Although the pumping feature increases the accuracy of the measurement, it must be noted that the passage of intense rapidly varying nonlinear internal waves present conditions that out-pace the ability of these instruments to respond. The thermal mass of the conductivity cell means that the cell and the water pumped in can have differing temperature, so that the water in the cell being analyzed for conductivity can have unknown temperature (to a small degree) (see Sec. II B). The mooring was located at $39.0250^{\circ}$ latitude, $-73.0668^{\circ}$ longitude, in an average water depth of $86.1 \mathrm{~m}$, and the mean instrument depths were 13.6, 16.6, 20.2, 25.1, 32.0, $39.1,47.1,56.1,65.1$, and $73.9 \mathrm{~m}$. The mooring on which the instruments were placed was very taught and thus "blow down" 
from currents was minimal, resulting in maximum instrument depth excursions of less than a meter, and maximum horizontal excursions of less than $15 \mathrm{~m}$. In addition, the mooring had a near bottom mounted ADCP to monitor currents. The ADCP provided observations of horizontal current starting at $68 \mathrm{~m}$ depth and proceeding in $4 \mathrm{~m}$ bins up to the surface.

Figure 1 shows the profiles of mean $T, S$, sound speed, buoyancy frequency, potential density, and potential soundspeed gradient, compared to the average of 15 CTD casts taken within $8.5 \mathrm{~km}$ of the mooring. The first four CTD profiles were taken on August 4, 5, 6, and 13, and the remaining 11 casts were obtained between 25 and 28 August. Given the intense variability of this region, the comparison between the mooring average and the CTD average is reasonable. A very strong pycnocline is observed between 10 and $30 \mathrm{~m}$ depth, giving rise to a maximum buoyancy frequency of over $30 \mathrm{cph}$. Additionally of note the potential sound-speed gradient is negative in the upper half of the water column and positive in the lower half. The conditions in the lower waters are caused by an underlying layer of warm, salty, offshore water penetrating onto the shelf, a feature sometimes referred to as the foot of the shelf/slope front.

\section{A. Isopycnal analysis}

Potential density (Talley et al., 2011) referenced to 40 decibar $(\mathrm{db})$ pressure $\left[\sigma_{40}(z(t), t)\right]$ was computed from the
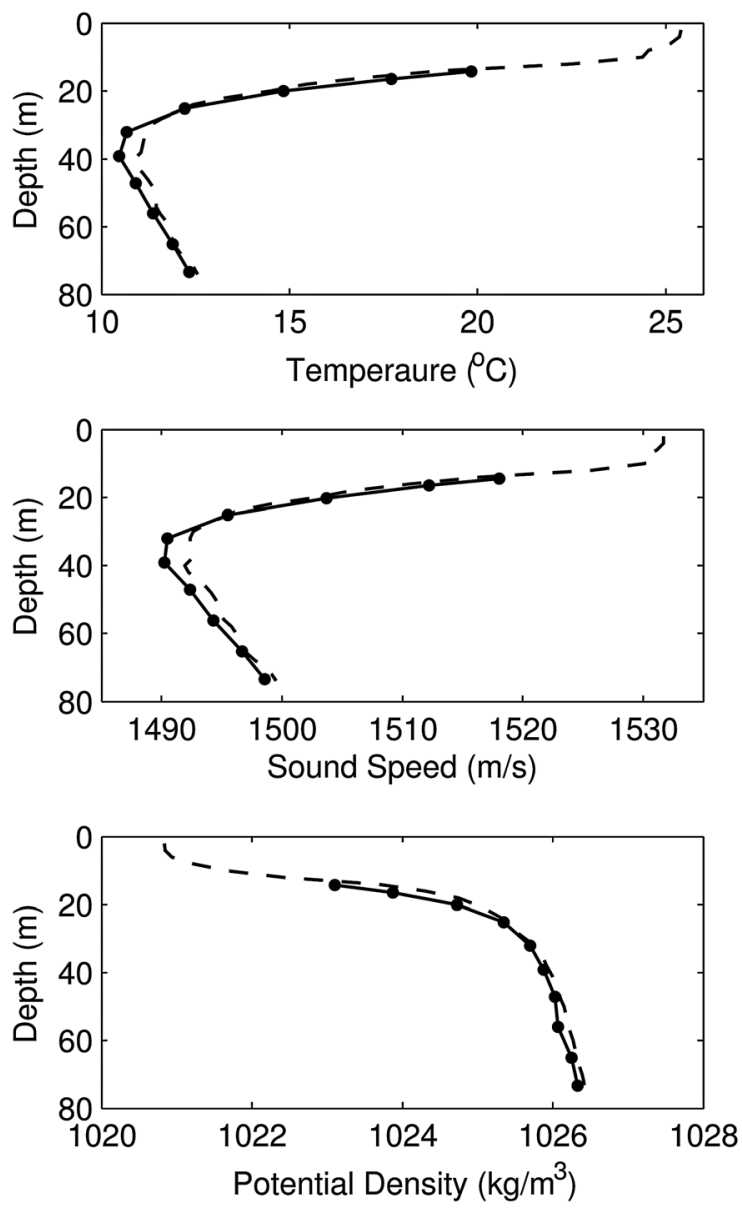

moored $T, S$, and $P$ time series, where $z(t)$ represents the time variable depth of the instruments due to mooring motion. From the computed potential density values, $\sigma_{40}(z(t), t)$, the depths for 11 isopycnals $\left[z\left(t, \sigma_{40}\right)\right]$, between 1025.3 and $1026.05 \mathrm{~kg} / \mathrm{m}^{3}$ (see Table I) were tracked using depth-linear interpolation. Potential density is needed in this analysis because internal-wave vertical lifting of density surfaces is accompanied by an adiabatic change in density (Dzieciuch et al., 2004). The $40 \mathrm{db}$ reference was chosen because it is roughly at the center of the water column and results for this reference are almost indistinguishable from those using the more traditional $0 \mathrm{db}$ reference. ${ }^{2}$ The spicy $T$ and $S$ values on the isopycnals $\left[T\left(t, \sigma_{40}\right)\right.$, and $\left.S\left(t, \sigma_{40}\right)\right]$ were obtained by linear interpolation of the original $T$ and $S$ data onto the computed isopycnal depth, $z\left(t, \sigma_{40}\right)$. As a measure of the accuracy to which the isopycnal depth could be tracked, the spicy $T\left(t, \sigma_{40}\right)$, and $S\left(t, \sigma_{40}\right)$ values were used to recompute potential density and the rms difference between this recomputed density and the target density gives the error (see Table I, third column). The small density errors ranging from 0.021 to $0.002 \mathrm{~kg} / \mathrm{m}^{3}$, show that the isopycnal tracking is quite good, even though simplistic linear interpolation was used. The isopycnal depth error can be estimated from the density error by dividing by the mean density gradient; these values are shown in Table I, fourth column. The small average depth errors ranging from 0.22 to $0.16 \mathrm{~m}$ again show that
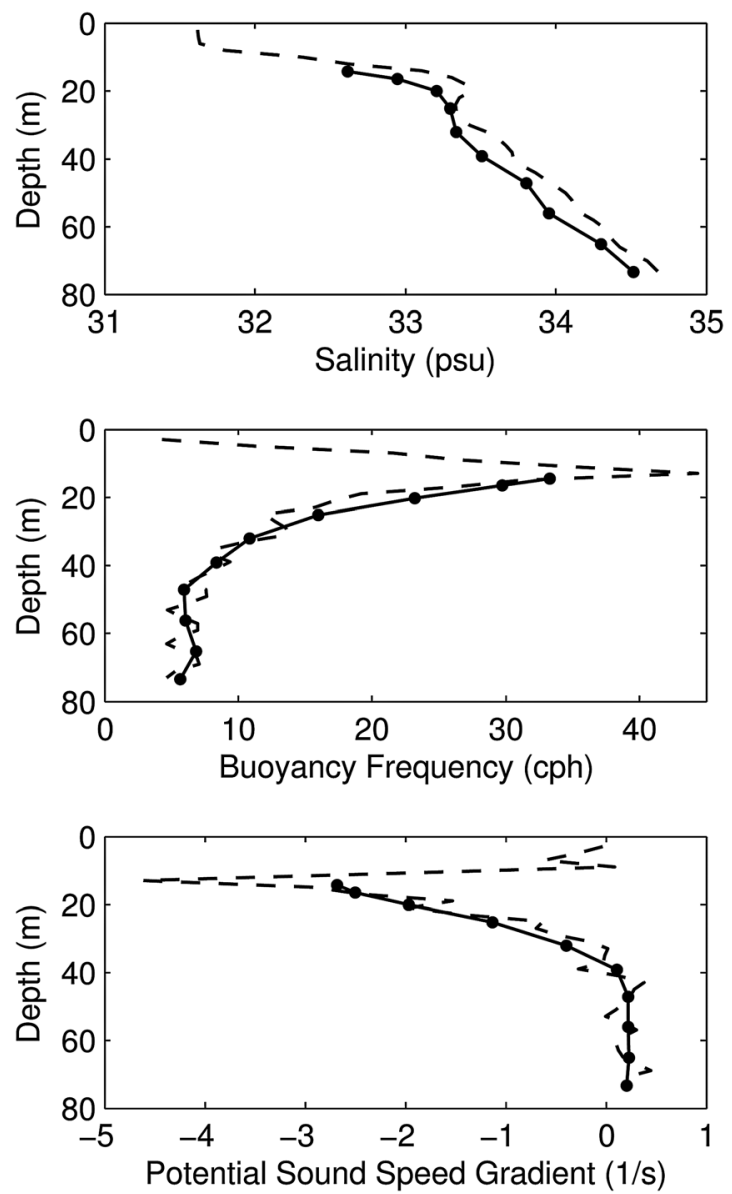

FIG. 1. Mean profiles of temperature, salinity, sound speed, buoyancy frequency, potential density, and potential sound-speed gradient (solid curves with points). Values from the average of 15 nearby CTD casts are shown with dashed curves (see the text). 
TABLE I. Some observed isopycnal statistics.

\begin{tabular}{|c|c|c|c|c|c|c|}
\hline$\sigma_{40}\left(\mathrm{~kg} / \mathrm{m}^{3}\right)^{\mathrm{a}}$ & $\langle z\rangle(\mathrm{m})^{\mathrm{b}}$ & $\delta \sigma_{40}\left(\mathrm{~kg} / \mathrm{m}^{3}\right)^{\mathrm{c}}$ & $\delta \sigma_{40} /\left(d \bar{\sigma}_{40} / d z\right)(\mathrm{m})^{\mathrm{d}}$ & $\delta T_{\mathrm{rms}}\left({ }^{\circ} \mathrm{C}\right)^{\mathrm{e}, \mathrm{f}}$ & $\delta S_{\mathrm{rms}}(\mathrm{psu})^{\mathrm{f}, \mathrm{g}}$ & $\delta c_{\mathrm{rms}}(\mathrm{m} / \mathrm{s})^{\mathrm{f}, \mathrm{h}}$ \\
\hline 1025.3 & 23.9 & 0.021 & 0.20 & 0.67 & 0.18 & 2.41 \\
\hline 1025.4 & 25.3 & 0.017 & 0.22 & 0.67 & 0.17 & 2.46 \\
\hline 1025.5 & 27.0 & 0.014 & 0.20 & 0.65 & 0.17 & 2.38 \\
\hline 1025.6 & 29.3 & 0.010 & 0.18 & 0.62 & 0.16 & 2.30 \\
\hline 1025.7 & 32.2 & 0.007 & 0.19 & 0.57 & 0.14 & 2.10 \\
\hline 1025.8 & 35.8 & 0.005 & 0.16 & 0.49 & 0.12 & 1.82 \\
\hline 1025.85 & 37.9 & 0.004 & 0.16 & 0.45 & 0.11 & 1.65 \\
\hline 1025.9 & 40.1 & 0.003 & 0.16 & 0.40 & 0.10 & 1.49 \\
\hline 1025.95 & 42.7 & 0.003 & 0.16 & 0.36 & 0.09 & 1.34 \\
\hline 1026.0 & 46.0 & 0.002 & 0.16 & 0.32 & 0.08 & 1.18 \\
\hline 1026.05 & 50.4 & 0.002 & 0.16 & 0.30 & 0.07 & 1.08 \\
\hline
\end{tabular}

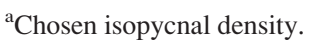

${ }^{\mathrm{b}}$ Mean isopycnal depth.

${ }^{c}$ rms uncertainty in the linear isopycnal tracking (see the text for details).

${ }^{\mathrm{d}}$ Depth error associated with the isopycnal tracking error.

${ }^{\mathrm{e}}$ Along the isopycnal rms potential temperature.

${ }^{\mathrm{f}}$ The rms values given in these last three columns have contributions from frequencies between $f$ and $N_{m}$, and corrections have been applied to remove the measurement noise at high frequency (see the text).

${ }^{\mathrm{g}}$ rms salinity variation along the isopycnal.

${ }^{\mathrm{h}}$ Sound-speed fluctuation along the isopycnal (spice).

the isopycnal tracking is working quite well. The specific range of densities, $1025.3-1026.05 \mathrm{~kg} / \mathrm{m}^{3}$ was chosen because these isopycnals remained in the moored array aperture over most of the 35 days of the measurements. A significant change in the background conditions, and a large storm event (tropical storm Ernesto) over the last 7 days of measurements caused some serious issues with isopycnal tracking, and thus the primary focus of this study is over the more stable 28 days initial period.

A few more words should be said about the linear depth interpolation method as it relates to the spiciness measurements. It is well known that the vertical structure of spicy or intrusive features can be quite detailed, with many length scales and with fluctuating gradients (Gregg and Cox, 1972; Munk, 1981). Our measurements, however, are not made in sufficient vertical resolution to resolve this nonlinear depth behavior. Therefore, the adopted linear interpolation approach only quantifies the large vertical scale spice structure, and must be considered a lower bound on the magnitude of the spice variations. The vertical correlation functions for the spicy temperature fluctuations observed in this experiment are presented in Sec. IV B of this paper and it is found that these functions are quite smooth. This result and the fact that the isopycnals are tracked rather accurately (Table I, third column) suggests that the resolved large scale structure may, in fact, be dominant in this particular case.

Figure 2 shows the observed mooring depth-time series of potential density $\sigma_{40}(z(t), t)$ and salinity $S(z(t), t)$, with a few isopycnal depths $z\left(t, \sigma_{40}\right)$ superimposed. The density field is seen to have a complex structure with variations due to changing background conditions, and energetic displacements due to internal waves and tides. The salinity field shows complex structure as well and the salinity variation along isopycnals (spice) is evident. In addition, in the salinity record the aforementioned change in the background thermohaline structure after day 28 is evident.
Figure 3 shows some examples of the derived isopycnal displacements, $z\left(t, \sigma_{40}\right)$, demonstrating periods of intense high frequency internal waves and periods of relative quiet with regard to these waves. In referring to the high frequency waves the term nonlinear waves will be used without proof of their nonlinearity as these waves have been extensively documented in the literature (Apel et al., 2007, and references therein). The upper panel of Fig. 3 shows the most energetic nonlinear wave train observed over the 35 day period.

This wave sequence shows the typical bore-like structure followed by a complex assemblage of over 10 internal solitary waves with peak-to-peak isopycnal displacement fluctuations of $10-20 \mathrm{~m}$. The lower panel of Fig. 3 shows a period in which the diffuse/broadband background internalwave field is evident, and here the isopycnal displacement fluctuations are much more modest and of order $5 \mathrm{~m}$ (peakto-peak).

\section{B. Spice analysis}

Internal-wave displacements create one form of soundspeed perturbation, with advection of density compensated $T$ and $S$ (spice) creating another. One can write

$$
\frac{\Delta C}{C}=a \Delta T+b \Delta S, \quad \frac{\Delta \rho}{\rho}=-\alpha \Delta T+\beta \Delta S,
$$

where typical values of the constants (which depend on pressure, $T$, and $S$ ) are (Munk, 1981)

$$
\begin{aligned}
& a=2.0 \times 10^{-3}{ }^{\circ} \mathrm{C}^{-1}, \quad b=0.74 \times 10^{-3} \mathrm{PSU}^{-1} \\
& \alpha=0.25 \times 10^{-3}{ }^{\circ} \mathrm{C}^{-1}, \quad \beta=0.75 \times 10^{-3} \mathrm{PSU}^{-1} .
\end{aligned}
$$

Thus, it is seen that along an isopycnal, temperature and salinity anomalies must be compensating, and hence the variation between cold and fresh and hot and salty along 

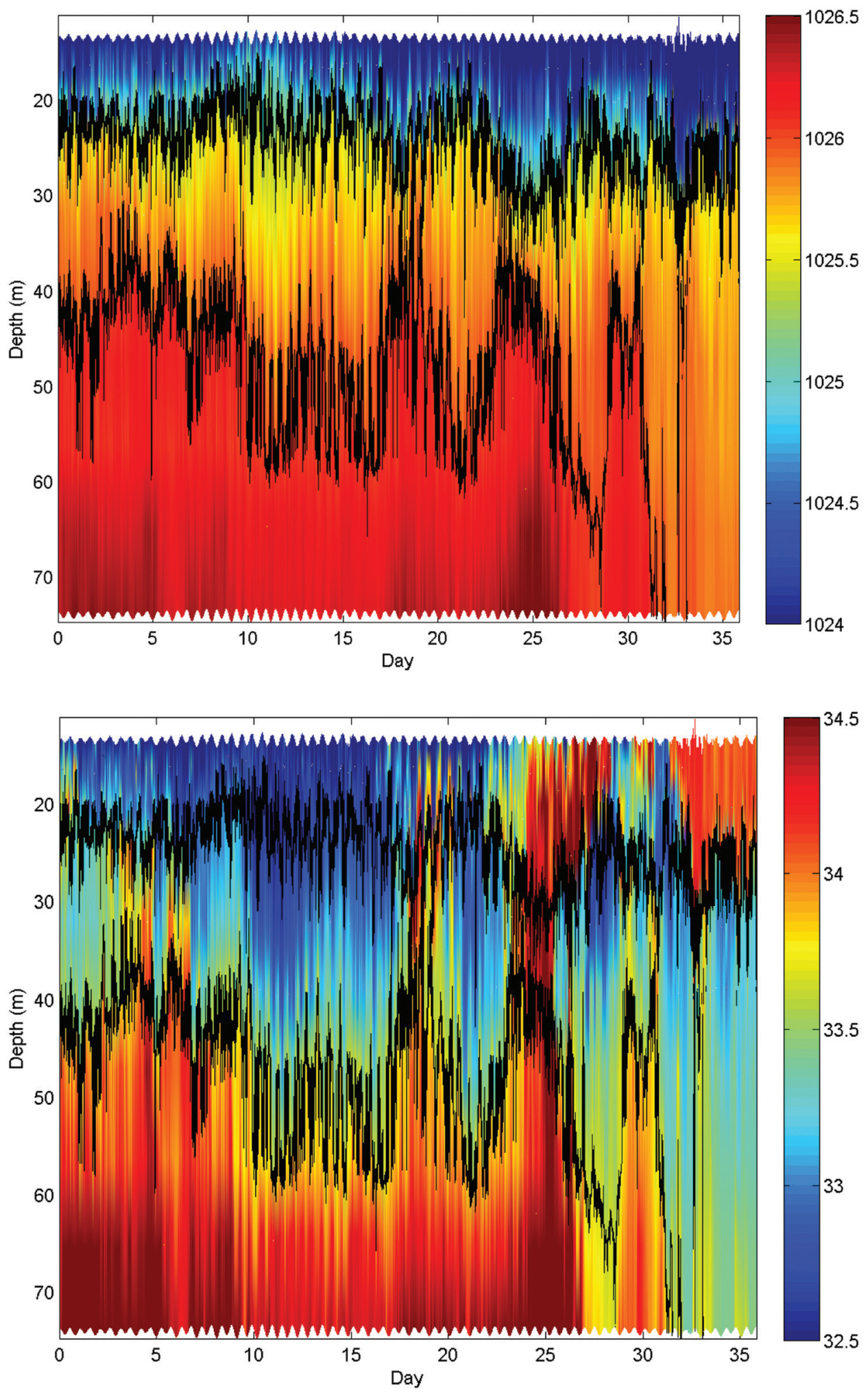

FIG. 2. Depth time series of potential density ( $\sigma_{40}$, upper panel), and salinity (lower panel). Superimposed on both plots are the isopycnal depths for densities of 1025.3 and $1026 \mathrm{~kg} / \mathrm{m}^{3}$. The zero of the time axis is 31 July 2006, 21:27:00 GMT.

isopycnals was termed spiciness (Munk, 1981). In addition it can be seen from Eq. (2) that for every degree of temperature change along an isopycnal there is an $\sim 3 \mathrm{~m} / \mathrm{s}$ change in sound speed; this is the spicy sound-speed effect. The spicy $T-S$ structure rides along with the internal-wave vertical isopycnal displacements, thus to quantify the spicy sound-speed effect alone this depth dependence must be eliminated. The spicy sound-speed fluctuations are thus computed using the mean depth of the isopycnal (Dzieciuch et al., 2004); this quantity is called $c_{S}\left(t, \bar{z}, \sigma_{40}\right)$. An example of the isopycnal $\sigma_{40}=1025.8 \mathrm{~kg} / \mathrm{m}^{3}$ time series of spicy sound-speed fluctuation, $c_{S}\left(t, \bar{z}, \sigma_{40}\right)$, as well as $T$ and $S$ variation, are shown in 

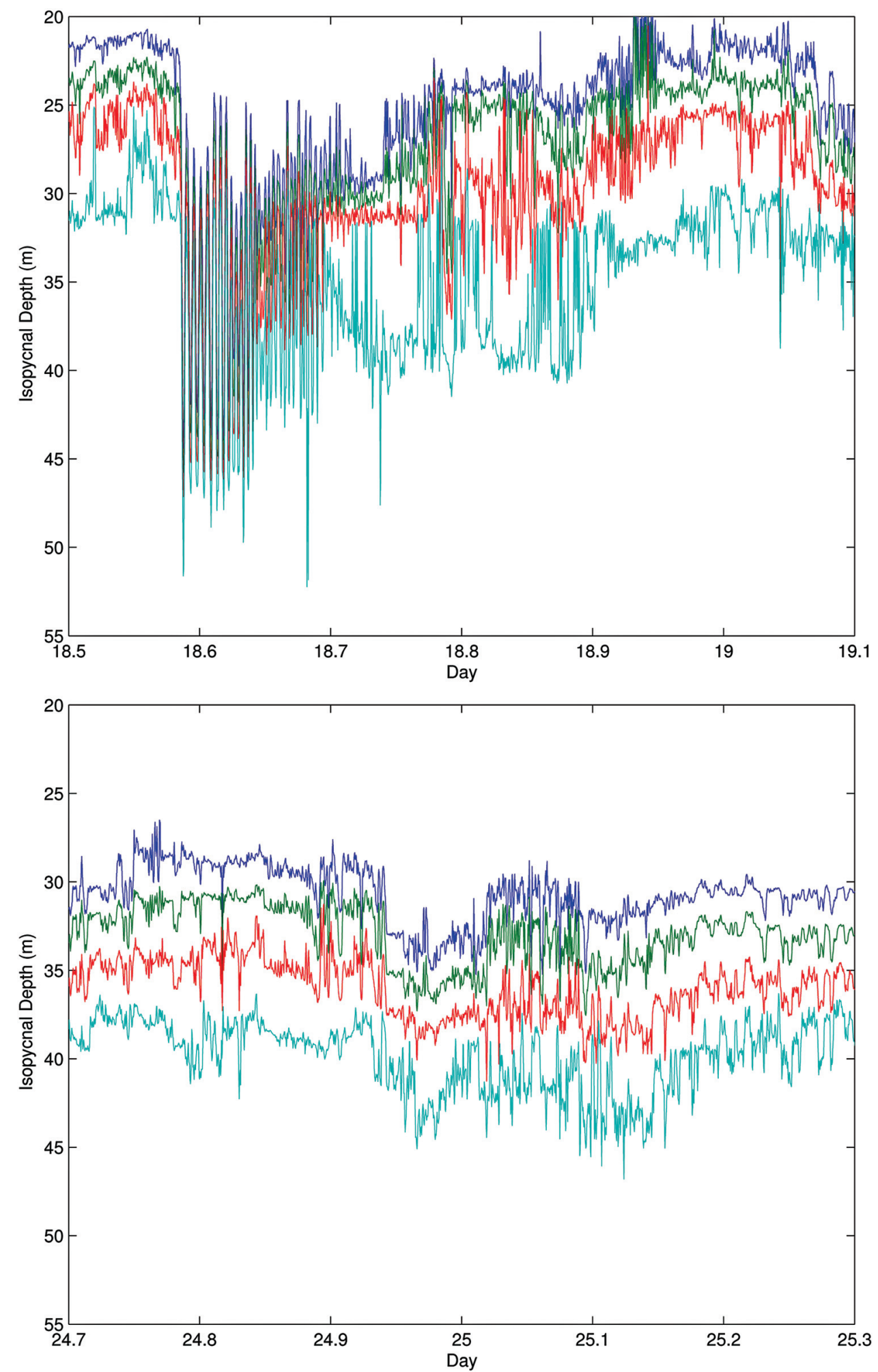

FIG. 3. Examples of isopycnal displacements for potential density values of $1025.3,1025.5,1025.7$, and $1025.9 \mathrm{~kg} / \mathrm{m}^{3}$. The upper panel shows a period in which nonlinear internal waves dominate the fluctuations, whereas the lower panel shows a period in which nonlinear waves and random waves have more equal contributions. The zero of the time axis corresponds to 31 July 2006, 21:27:00 GMT.

Fig. 4; the density compensating effect of $T$ and $S$ is clearly seen in the strong correlation of these time series. Time series of spicy sound-speed corresponding to the time range of Fig. 3 are shown in Fig. 5. The large spicy sound-speed variations observed in the nonlinear internal-wave packet (upper panel) are quite surprising, and if it were to be believed that internal-wave horizontal currents are advecting the spicy structure, the $6 \mathrm{~m} / \mathrm{s}$ swings in sound speed suggest extremely large temperature variability along the isopycnal $\left(\sim 2{ }^{\circ} \mathrm{C}\right)$. As previously noted, the SBE37 SMP sensors have difficulty obtaining accurate salinities when the temperature is rapidly varying, as is the case in this nonlinear internal- 

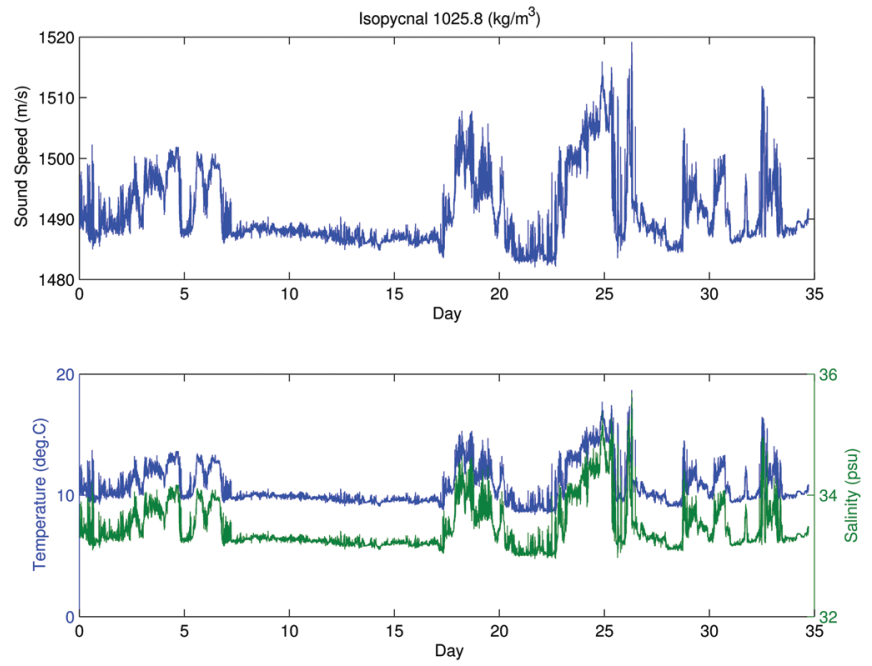

FIG. 4. Fluctuations of spicy sound speed, $c_{S}$ (upper panel) and temperature and salinity (lower panel) along the isopycnal $1025.8 \mathrm{~kg} / \mathrm{m}^{3}$. The zero of the time axis corresponds to 31 July 2006, 21:27:00 GMT.
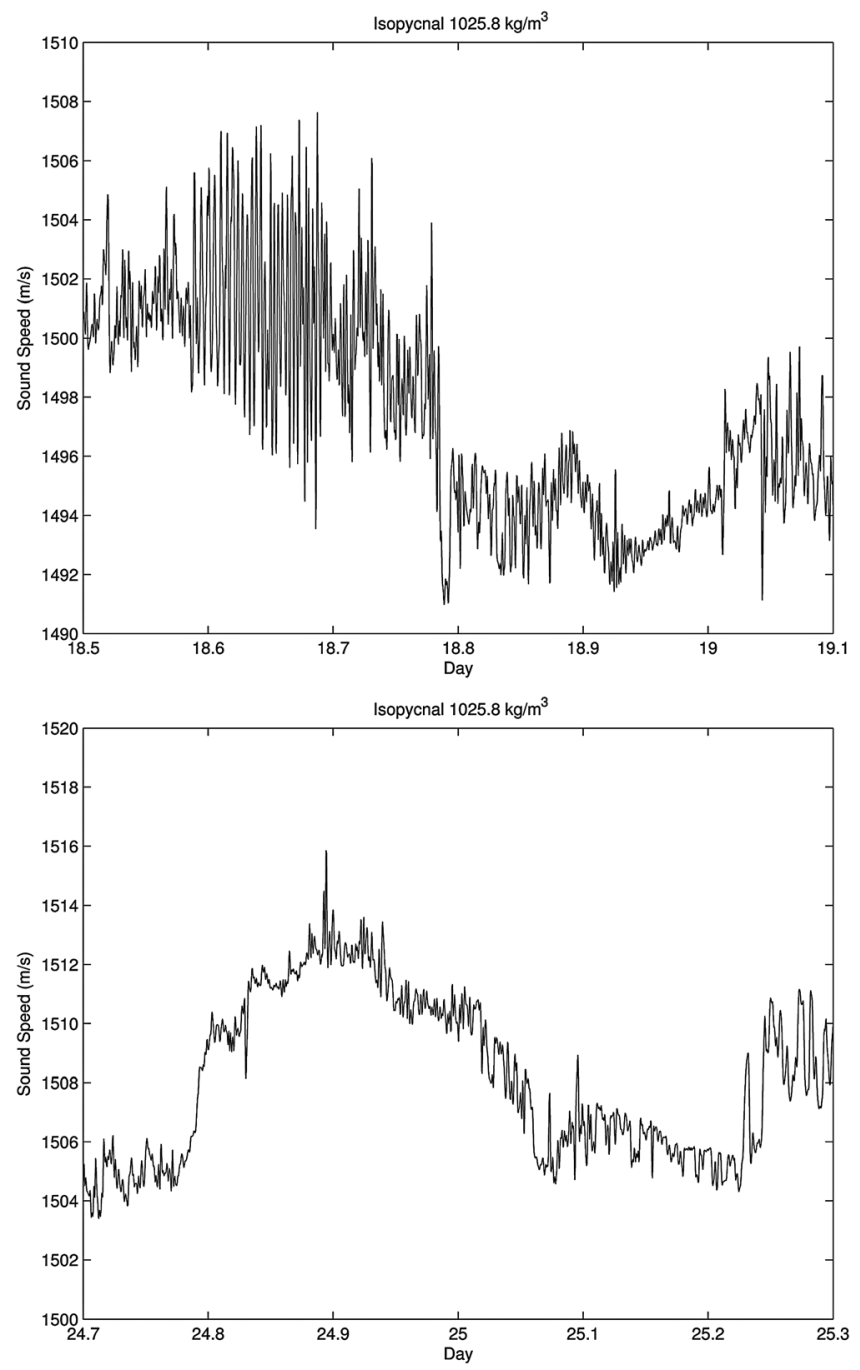

FIG. 5. Fluctuations of spicy sound speed $\left(c_{S}\right)$ for the time regions corresponding to Fig. 3 . The zero of the time axis corresponds to 31 July 2006, 21:27:00 GMT. wave packet, thus it is highly likely that this variability is an artifact of the limited response of the instruments. Future measurements with moored or towed, fast-response CTDs would be needed to resolve this issue, but in the present analysis these high frequency spice fluctuations will be treated as unphysical noise.

\section{FREQUENCY SPECTRA AND ANALYSIS OF VARIANCE}

In this section frequency spectra for internal waves and spicy thermohaline structure are presented to give a sense of the time scales involved with these processes. In addition, the depth dependence of internal-wave and spicy structure are of fundamental importance to acoustics. Here the depth dependence of the sound-speed variance is quantified in order to understand the regions in the water column in which acoustic scattering may be largest. The analysis starts with the internal-wave field.

\section{A. Internal waves}

The observed time series of isopycnal displacements, $z\left(t, \sigma_{40}\right)$, were used to compute frequency spectra that facilitate a separation of the diffuse broadband internal-wave field fluctuation statistics from the internal tide and the nonlinear internal-wave field variability. Before the spectral analysis was performed the isopycnal displacement time series were low-pass filtered using a four-pole Butterworth digital filter to eliminate signals with frequencies greater than $40 \mathrm{cph}$ (close to the maximum buoyancy frequency). To minimize side band leakage from the energetic low frequencies, spectragrams for the filtered isopycnals were computed using the multitaper method of Thompson (Percival and Walden, 1993; Thompson, 1982) with an estimated time-bandwidth product of 4 and a record length of $12 M_{2}$ tidal cycles or roughly 6.21 days. The spectragrams were averaged over the 28 day period using 1/2 overlapping segments to provide a power spectral density (PSD) estimate for each of the 11 isopycnal densities that is denoted by $S_{\xi}\left(\omega, \bar{z},\left(\sigma_{40}\right)\right)$, where $\bar{z}\left(\sigma_{40}\right)$ is the mean depth of the isopycnal, $\sigma_{40}$. Figure 6 shows the average PSD over all 11 isopycnals. The displacement spectrum is seen to have a strong semidiurnal tide line, a power law region for frequencies between $f$ and roughly 24 cycles per day (cpd), and an enhanced high frequency region. Here it is proposed that the power law behavior be associated with diffuse internal waves (DIW) and the enhanced high frequency region be connected with nonlinear internal waves (NLIW), such as internal solitary waves as shown in Fig. 3(upper panel). This separation is reasonable, as random internal waves in the ocean follow power law spectra (Munk, 1981), and nonlinear waves generally have high frequencies (Apel et al., 2007). The semidiurnal line at a frequency of $1.9324 \mathrm{cpd}$ is clearly associated with internal tides (IT). As Fig. 3 shows there is certainly some nonlinearity associated with the internal tide, but this effect will be ignored in the nonlinear wave statistics as it appears to be weak on average as strong tidal harmonics are not clearly evident in the spectra (a small second harmonic peak of the semidiurnal frequency is marginally visible). 

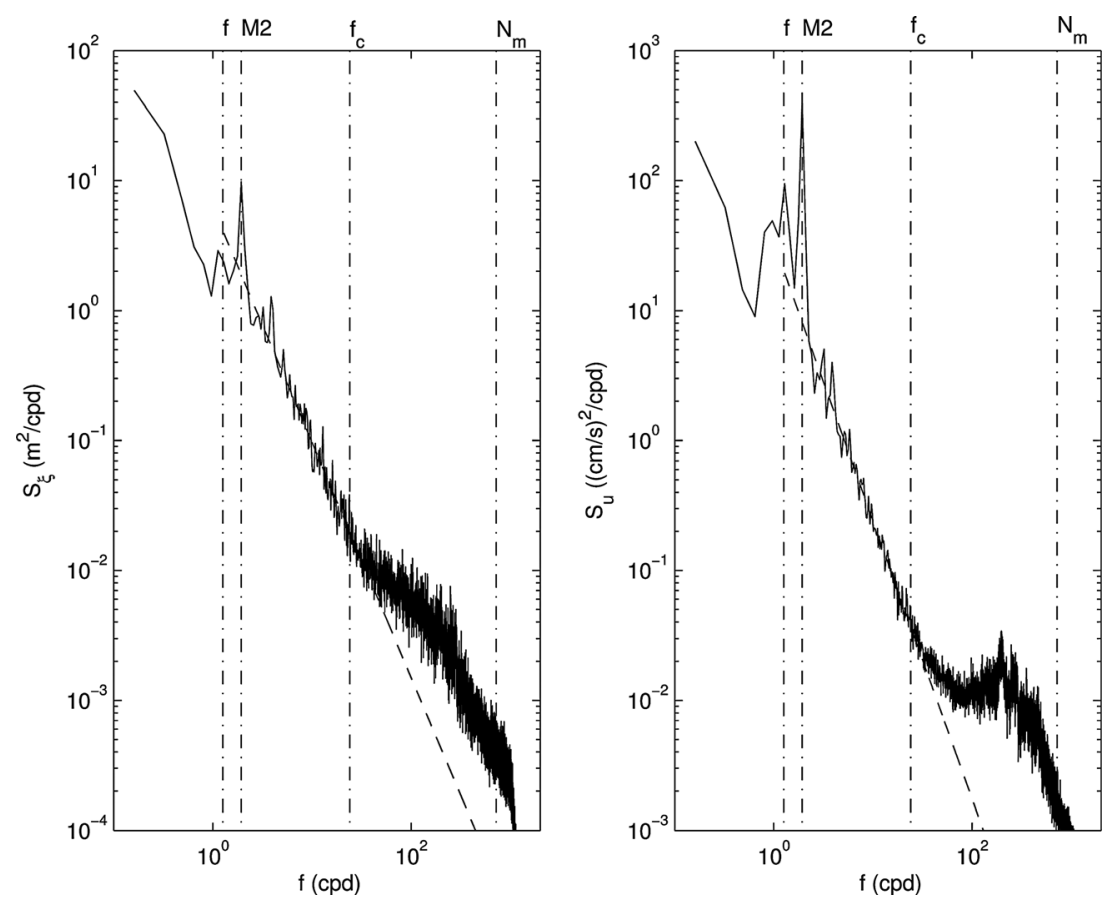

FIG. 6. Frequency spectra of displacement averaged over 11 isopycnals (left) and horizontal current averaged over a similar depth region (right). Vertical dashed-dotted lines denote the Coriolis frequency $f$, the semidiurnal tide frequency $M 2$, the cutoff frequency $f_{c}$, and the maximum buoyancy frequency $N_{m}$. Diffuse broadband internal waves are seen to dominate for frequencies between $f$ and roughly 24 cpd, whereas nonlinear internal waves dominate at higher frequencies. A power-law fit to the diffuse broadband wave displacements gives an exponent of $p=-1.81 \pm 0.25$, whereas the fit for the horizontal currents gives a power-law exponent of $p=-2.10 \pm 0.19$. The high frequency cutoff at $1000 \mathrm{cpd}$ is due to a low pass filter applied to the data.

As a further consistency check on the adopted isopycnal tracking method (and thus the displacement frequency spectrum for which our analysis is based), the frequency spectrum of horizontal currents is computed using the ADCP observations. Except near the inertial frequency, these two spectra should have identical form. To be consistent with the calculation of the displacement spectrum, the horizontal current spectrum was averaged over the same depth region as the 11 isopycnals. Figure 6 shows the two spectra, which compare fairly well. In the power law region, fits to the spectra between $2 f$ and $24 \mathrm{cpd}$ give exponents of $p=-1.81 \pm 0.25$ for the displacement spectrum and $p=-2.10 \pm 0.19$ for the current spectrum. The error bars on the power law exponents represent Gaussian error 95\% confidence intervals, and thus the two values are consistent statistically at this level. Both spectra show enhanced energy at frequencies in excess of $1 \mathrm{cph}$, and they show a strong semidiurnal tide line.

These spectral estimates can be compared to two other analyses from the Mid-Atlantic Bight. First Yang and Yoo (1999) present frequency spectra of sound-speed perturbations estimated from $2 \mathrm{~h}$ of yo-yo CTDs in which nonlinear internal waves do not obviously appear in the data. This study is clearly limited because (1) the short time duration of the data and (2) the sound-speed data have internal waves and spice mixed together. This study finds a spectral slope of -1 , in the frequency range $0.5-15 \mathrm{cph}$, which is very different from the value derived from the present analysis. Another result, appearing in Apel et al. (1997), presents diffuse internal-wave frequency spectra derived from $6 \mathrm{~h}$ sections of ADCP data in which nonlinear internal waves do not obviously appear in the records. These results have much better statistics, having been derived from multiple $6 \mathrm{~h}$ sections, and they represent internal waves and not spice. Here the spectral slope is estimated to be $-1.5 \pm 0.1$ in the frequency band $0.4-4 \mathrm{cph}$, which is roughly consistent statistically with the present value of $p=-1.81$ \pm 0.25 , derived from the frequency band $f$ to $1 \mathrm{cph}$.

Of fundamental interest to ocean acousticians are the relative strengths of the displacement fluctuations due to diffuse, tidal, and nonlinear internal waves. Because these processes are statistical in nature a statistical metric is sought to quantify this relationship. rms statistics are useful for the diffuse and tidal components because these waves are always present in the record. On the other hand, rms statistics do not lend themselves well to quantifying the nonlinear waves because these waves are non-Gaussian and strongly episodic: Be that as it may, the rms metric is utilized here because it is a well-understood statistic: The reader must therefore keep this in mind when interpreting the nonlinear wave estimates.

The spectrum will serve as an important tool in this analysis. Here the assumption is made that only diffuse internal waves and internal tides are present in the frequency spectral range between $f$ and $f_{c}=24 \mathrm{cpd}$, whereas nonlinear waves (such as internal solitary waves) are the dominant contributions between $f_{c}$ and the maximum buoyancy frequency $N_{m}=1000 \mathrm{cpd}$. Further, it is assumed that the internal-tide contribution is linear and phase locked, and thus this contribution can be estimated by carrying out a least-square tidal fit using four semidiurnal constituents [S2, M2, N2, and K2 (Talley et al., 2011)]. Clearly the internal tide has some nonlinearity (i.e., Fig. 3), and previous studies have shown some degree of phase variability of the internal tide (Colosi et. al., 2001); this effect is simply ignored to obtain a first-order estimate of the internal-tide strength. The rms internal-tide displacement fluctuation for the 11 

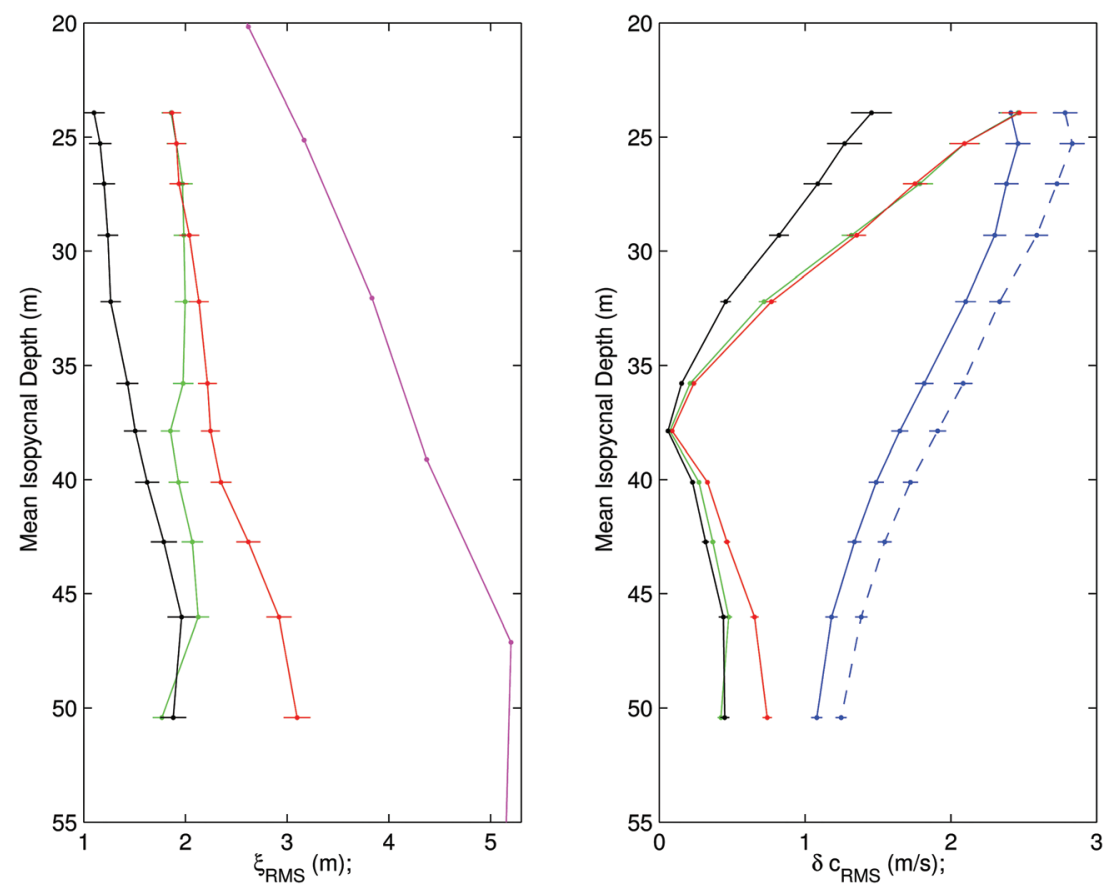

FIG. 7. Rms displacement (left panel) and sound speed (right panel) caused by internal waves and intrusive fine structure (spice). Horizontal lines correspond to rms estimation uncertainties. Black curves correspond to internal tides, green curves are for nonlinear internal waves, and red curves are for diffuse internal waves. The magenta curve in the left-hand panel is a WKB estimate based on $\xi_{\text {rms }}=\xi_{0} \sqrt{N_{0} / N(z)}$, where $N(z)$ is obtained from Fig. 1 . The blue dashed curve in the right-hand panel shows the rms sound-speed fluctuation from spice in the frequency band between $f$ and $N_{m}$, and the solid blue shows the rms sound-speed fluctuation from spice after removing the error due to the high frequencies (see the text).

isopycnals obtained in this way is shown in Fig. 7. To estimate the displacement variance contributions from the diffuse internal waves and the nonlinear internal waves, the spectrum of the detided records are computed and denoted by $\hat{S}_{\xi}(\omega, \bar{z})$. The individual variance estimates are obtained by integrating over the spectrum or

$$
\begin{aligned}
& \left\langle\xi^{2}\right\rangle_{D I W}(\bar{z})=\int_{f}^{f_{c}} \hat{S}_{\xi}(\omega, \bar{z}) d \omega+\gamma^{2}, \\
& \left\langle\xi^{2}\right\rangle_{N L I W}(\bar{z})=\int_{f_{c}}^{N_{m}} \hat{S}_{\xi}(\omega, \bar{z}) d \omega-\gamma^{2},
\end{aligned}
$$

where $\gamma^{2}$ is a correction due to the fact that the diffuse internal-wave spectrum is expected to extend into the high frequency region between $f_{c}$ and $N_{m}$. This correction is computed by doing a power law fit to the detided low frequency spectrum between $f$ and $f_{c}$ (see Fig. 6) and integrating the expected variance from this power law in the high frequency region between $f_{c}$ and $N_{m}$. The nonlinear contribution, however, needs one further correction. It must be taken into account that the nonlinear internal waves are waves of depression. As such, the spectrum measures the zerocentered fluctuation of the wave (such as $\cos \omega t$ ) where as the nonlinear wave has a form more akin to $(1+\cos \omega t)$; the mean square of the former being $1 / 2$ and of the latter $3 / 2$ giving a factor of 3 . The rms diffuse internal-wave contribution and the nonlinear wave contribution corrected for the factor of $\sqrt{3}$ are plotted in Fig. 7 .

Figure 7 shows the vertical distribution of rms internalwave displacement for the internal tide, the diffuse waves, and the nonlinear waves. The internal tide and diffuse waves show an increasing fluctuation from 24 to $46 \mathrm{~m}$ depth, which is consistent in shape with the Wentzel-Kramers-Brillouin (WKB) prediction $\xi_{\mathrm{rms}}=\xi_{0} \sqrt{N_{0} / N(z)}$ (Munk, 1981). In Fig. 7, the WKB curve is computed using $\xi_{0}=7.3 \mathrm{~m}$, and $N_{0}=3 \mathrm{cph}$, which are values consistent with the Garrett-Munk deep-water internal-wave model; a value of $\xi_{0}$ more consistent with the observations is $4.5 \mathrm{~m} .^{3}$ The diffuse internal waves are seen to closely follow the shape of the WKB result, which is understandable as the diffuse waves have contributions from high internal-wave modes where the WKB theory works best (see Sec. IV). The internal tide result does not follow the WKB result as well, as this fluctuation is dominated by the first few modes where WKB theory is not as accurate (again, see Sec. IV). The vertical distribution of the nonlinear internal-wave fluctuation shows values nearly independent of depth, likely due to the well-known low mode nature of these waves (Apel et al., 2007).

The rms sound-speed anomaly from the three types of waves at each mean isopycnal depth can be calculated using the following relation: $\delta c_{\text {rms }}(\bar{z})=\left|(d c / d z)_{p}(\bar{z})\right| \xi_{\text {rms }}(\bar{z})$. The results are plotted in Fig. 7(right-hand panel). The rms sound-speed fluctuations from internal waves diminish rapidly below the main thermocline where the sound-speed gradient goes through zero (see Fig. 1). The observed sound-speed fluctuation for the diffuse internal waves are quite large in the main thermocline, and exceed $2 \mathrm{~m} / \mathrm{s} \mathrm{rms}$. Below the thermocline values are typical of deep water environments and are less than $1 \mathrm{~m} / \mathrm{s}$ rms (Beron-Vera et al., 2003). 


\section{B. Spicy thermohaline structure}

Spectra were computed from the 11 spice sound-speed time series, $c_{S}\left(t, \bar{z}, \sigma_{40}\right)$, as described in Sec. II B, and the average spectrum is shown in Fig. 8; this spectrum is termed the spice spectrum. In the internal-wave band between $f$ and $N$ the spectra for internal-wave displacement (Fig. 6) is quite similar to the spice spectrum, although the semidiurnal tide line and the inertial peak blend together in the spice spectrum. The similarity of the low frequency portion of the spectra suggests that internal-wave horizontal advection of the $T / S$ structure along the isopycnal may be the dominant cause of the observed fluctuations on the mooring as is the standard view from the physical oceanographic literature (Phillips, 1971; Garrett and Munk 1971; Munk 1981). As previously mentioned, the large inferred spicy sound-speed fluctuations occurring during the passage of nonlinear internal waves are somewhat suspect, and thus the "high frequency" end of this spectrum with frequencies greater than $24 \mathrm{cpd}$, must be interpreted for now as noise. In the region between $2 f$ and $f_{c}$ the spice spectrum shows a power law behavior with a power law exponent $p=-1.73 \pm 0.20$ being somewhat smaller than the internal-wave exponent of $-1.81 \pm 0.25$. It is important to note that the prevailing view of spicy ocean features is that they are front-like (Ferarri and Rudnick, 2000), and therefore should have a spectral slope close to -2 (i.e., the spectral slope of a step function). The observed value with uncertainties, $p=-1.73 \pm 0.20$, is in fact quite consistent with the frontal view of spice. It should also be noted that the present analysis is focused on fluctuation in spice within the internalwave frequency band, but as is seen in Figs. 4 and 8 there are strong subinertial fluctuations in the spice.

To quantify spicy $T, S$, and sound-speed variations along the isopycnals, spectra of $T, S$, and sound speed were integrated from $f$ to $N_{m}$ to yield variances; rms values of $T, S$, and sound speed are shown in Table I and rms sound speed is also

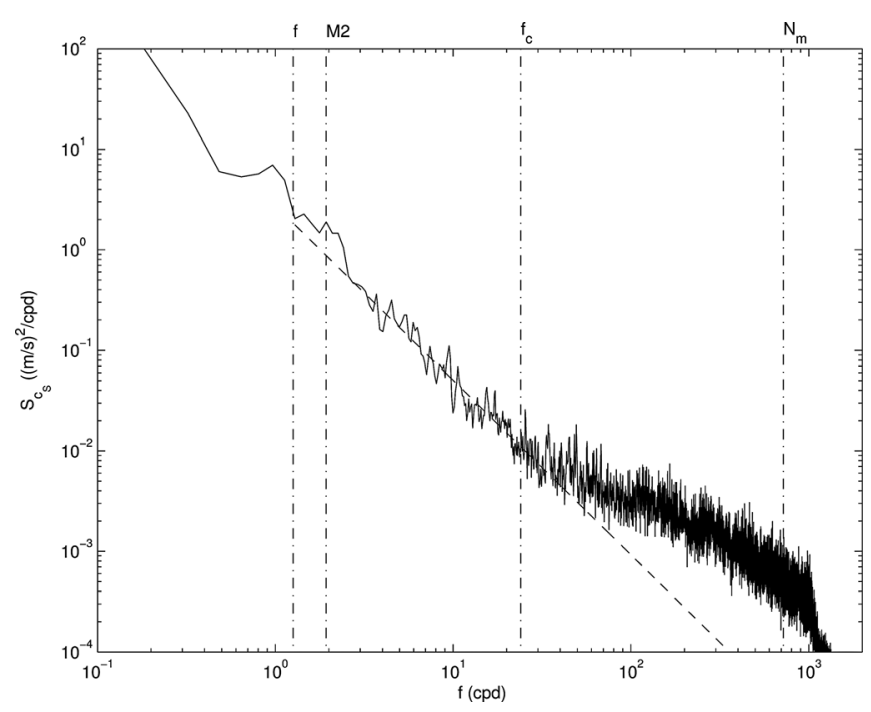

FIG. 8. Average frequency spectrum of sound-speed fluctuations along the 11 isopycnals due to spice. Vertical dashed-dotted lines denote the Coriolis frequency $f$, the semidiurnal tide frequency $M 2$, the cutoff frequency $f_{c}$, and the maximum buoyancy frequency $N_{m}$. A power-law fit to the low frequency end of the spectrum yields an exponent of $-1.73 \pm 0.20$. plotted in Fig. 7(right-hand panel). The rms values in Table I and the solid blue curve in Fig. 7 have been corrected to remove the high frequency instrument noise in the following way. First the spectrum is integrated from $f$ to $f_{c}$ and then a high frequency contribution is added to that by extending a power law fit from $f_{c}$ to $N_{m}$ as was done for the diffuse internal-wave displacements [see Eq. (3)]. Figure 7, in fact, shows two spice curves; the dash curve in which the variance is obtained by integrating the entire spectrum (including the questionable high frequencies), and a solid curve, which has the high frequency correction as described previously. Here it is seen that the questionable high frequency spice variations do not contribute significantly to the over all variance. It is also seen that the spice effects are larger in the upper ocean where spice generating mixing is more pronounced (Ferarri and Rudnick, 2000). Last from Table I it is evident that the sound-speed fluctuation is driven primarily by the temperature fluctuations along the isopycnals with an expected $3.7 \mathrm{~m} / \mathrm{s} /{ }^{\circ} \mathrm{C}$.

These results show that in this particular experiment and location the dominant source of rms sound-speed fluctuation is spice, and thus future analysis will be required to see how representative the results are. For example one may rightly ask, how do these results depend on location or time of year, as well as other oceanographic/atmospheric processes like eddies and storms? The present result suggests the importance of spicy structure in shallow-water acoustic propagation, and future studies will be required to better understand the associated acoustic scattering effects.

\section{VERTICAL STRUCTURE}

Having addressed the temporal nature of the soundspeed fluctuations and the vertical distributions of variance, an analysis of the vertical spatial structure is now presented. The nature of the vertical structure is acoustically significant because of the importance of vertical sound-speed gradients to acoustic propagation and scattering.

\section{A. Diffuse internal waves and internal tides}

In this section the vertical structure of the diffuse and tidal components of the internal-wave field are examined. The vertical structure of the nonlinear internal waves is of keen acoustical importance, but a detailed analysis of these episodic waves and their nonlinear dynamics is beyond the scope of the present paper.

In this analysis a useful metric of the vertical structure is the depth covariance function, which of course is closely related to the mode spectrum (more later). In computing the covariance functions for the diffuse internal waves and tides the 11 displacement records will be used with some modification. Regarding the internal tide, the tidal fits for each isopycnal will give the internal tide record at each mean depth, denoted by $\xi_{I T}(t, \bar{z})$. Regarding the diffuse internal waves it is seen that aside from the narrow semidiurnal tide band, these waves dominate between the Coriolis frequency $(f)$ and roughly $24 \mathrm{cpd}$. Therefore the diffuse internal-wave record to be used in the covariance analysis is the detided displacement record, bandpass filtered between $f$ and $24 \mathrm{cpd}$; this record is denoted by $\xi_{D I W}(t, \bar{z})$. The associated covariance matrices 
are therefore $C_{D I W}\left(z_{1}, z_{2}\right)=\left\langle\xi_{D I W}\left(t, \bar{z}_{1}\right) \xi_{D I W}\left(t, \bar{z}_{2}\right)\right\rangle$ and $C_{I T}\left(z_{1}, z_{2}\right)=\left\langle\xi_{I T}\left(t, \bar{z}_{1}\right) \xi_{I T}\left(t, \bar{z}_{2}\right)\right\rangle$, where the angular brackets represent averages over time. The rms error in the covariance estimates are obtained by computing the rms variation of the individual covariance estimates at each time around the mean value, and dividing by the square root of the number of independent samples. For the diffuse internal waves an independent sample is obtained roughly every 0.1 day, whereas for the internal tide a value of 0.5 day was adopted.

Before analyzing the observed covariances, a physical model in which these functions can be interpreted is presented. For the diffuse internal waves and the internal tide the linear dynamics approximation is useful and the displacement is written as a linear modal expansion. It is found that for the SW06 environment, the linear internal-wave modes have very little variation between the Coriolis frequency and 24 cpd (Colosi et al., 2012) and so the observations bandpass filtered in this range can be modeled as

$$
\xi(z, t)=\sum_{j=1}^{J} a_{j}(t) \phi_{j}(z),
$$

where $J$ is the maximum internal-wave mode number (assumed independent of frequency). The mode functions are derived for a resting, linear, flat-bottom ocean such that

$$
\frac{d^{2} \phi_{j}}{d z^{2}}+k_{j}^{2} \frac{N^{2}(z)-\omega^{2}}{\omega^{2}-f^{2}} \phi_{j}=0,
$$

where the modes have the normalization

$$
\int_{0}^{D}\left(N^{2}-\omega^{2}\right) \phi_{j}^{2} d z=1
$$

The mode approximation is certainly incorrect dynamically as any one of the three assumptions (resting, linear, and flat bottom) is likely violated for the SW06 environment, however, the main justification for using the modes is that they do form an orthonormal set. Using the modal expansion of Eq. (4) and assuming that the amplitudes of the modes are uncorrelated, the covariance matrix has the form

$$
\left\langle\xi\left(z_{1}\right) \xi\left(z_{2}\right)\right\rangle=\sum_{j=1}^{J} H(j) \phi_{j}\left(z_{1}\right) \phi_{j}\left(z_{2}\right),
$$

where $H(j)=\left\langle\left|a_{j}\right|^{2}\right\rangle$ is the mode spectrum; this is the equivalent of the Weiner-Khinchin relation.

\section{Estimation of the mode spectrum}

Here an inverse procedure for estimating a spectrum from observations of the covariance function is described. Equation (6) can be cast in matrix form, and accounting for measurement uncertainty the result is

$$
\mathbf{y}=\mathbf{E x}+\mathbf{n}_{\mathbf{y}}
$$

where $\mathbf{y}=\left\langle\xi\left(z_{1}\right) \xi\left(z_{2}\right)\right\rangle$ is a vector of observed covariances, $\mathbf{E}=\phi_{j}\left(z_{1}\right) \phi_{j}\left(z_{2}\right)$ is the matrix of mode shape products, $\mathbf{x}=H(j)$ is the mode spectrum vector, and $\mathbf{n}_{\mathrm{y}}$ is an uncer- tainty on the covariance estimates (e.g., due to sampling errors). If $M$ is the number of depth points, and $J$ is the number of internal-wave modes, then $\mathbf{y}$ and $\mathbf{n}_{\mathrm{y}}$ are vectors of length $M^{2}$, and $\mathbf{E}$ is an $M^{2} \times J$ matrix. In the present work $M=11$ (isopycnals) and $J$ values of 15 and 5 are used for the diffuse internal waves and internal tides, respectively. It should be re-iterated and stressed here that a specific mode set has been assumed and that the amplitudes of theses modes are assumed to be uncorrelated. Equation (6) could be modified so as to estimate not only the mode spectrum but also the cross mode coherences. These coherences can be important if the modes $\phi_{n}(z)$ are not the natural modes of the system or if there are, in fact, real correlations due to any number of geophysical processes. On the other hand, even in the case of strong cross-mode coherences, their contribution to the covariance function can often be small due to the fact that they have variable phase (Colosi and Morozov, 2009). Thus, in the interest of simplicity the complication of cross mode coherences is ignored.

There is one more factor contributing to the covariance function that needs to be considered and that is the effect of noise in estimating the isopycnal depth from the temperature, salinity, and pressure. For uncorrelated isopycnal noise the diagonals of the covariance matrix are the sum of the signal and noise variances, and the off-diagonal elements are simply that of the signal. Thus, a non-negative isopycnal displacement noise variance is included in the elements of the vector $\mathbf{y}$ corresponding to the diagonal of the instrument covariance matrix. Consequently to account for this effect on the right-hand side of Eq. (7), 11 isopycnal noise variance parameters, $\epsilon^{2}$ (one for every isopycnal) are appended to the vector of unknowns $\mathbf{x}$, and 11 extra columns are added to the matrix $\mathbf{E}$. The added columns in $\mathbf{E}$ are all zero except for a single 1 for each of the 11 rows that correspond to autocovariances. A few cautionary notes about the assumption of uncorrelated isopycnal noise and other errors must be made. It must be noted that because the "observations" of displacement are derived from observations of temperature, salinity, and pressure, the isopycnal measurement noise may have (unknown) large scale correlations. In the face of this unknown the best approach is to assume uncorrelated contributions, and judge the success of this assumption based on the quality of fit and the associated fit error bars: The fits are indeed found to be satisfactory. In addition, "representational" error due to the truncation of the model spectrum (i.e., choice of $J$ ) could be present, with short vertical decorrelation scales. After some experimentation with this, the error was judged to not be significant with the generous truncations used in the fits.

Equation (7) is an analog to power spectrum estimation. In order to utilize simple estimation techniques based on Gaussian statistics, some modification of Eq. (7) must be carried out as $\mathbf{x}$ is non-negative. The approach taken here is to provide a first-guess spectrum and noise variance, $\mathbf{x}_{0}$, and compute the corrections to this guess. The inverse so formulated is then of the form

$$
\delta \mathbf{y}=\mathbf{E} \delta \mathbf{x}+\mathbf{n}_{\mathbf{y}},
$$


where $\delta \mathbf{x}=\mathbf{x}-\mathbf{x}_{0}$ is the correction being sought and $\delta \mathbf{y}=\mathbf{y}-\mathbf{E} \mathbf{x}_{0}$ is the difference between the observations and the predicted values based on the guess. Note that the statistics of these corrections are probably not exactly Gaussian, but in practice the total estimates (guess plus correction) were never negative. Many estimation methods exist for non-negative quantities, including linear and quadratic programming, but the simplicity of least squares is attractive. A variety of simple least-squares techniques could be used to solve Eq. (8) including the singular value decomposition. The Gauss-Markov estimator is chosen here because of its clarity of interpretation and correspondence to weighted least squares. Importantly this method provides not only estimates of the corrections to the mode spectrum and the noise variance parameters but also their associated uncertainties and correlations (Munk et al., 1995). Using the Gauss-Markov method, the estimates of $\delta \mathbf{x}$ and its covariance $\mathbf{P}$ are

$$
\begin{aligned}
\delta \mathbf{x} & =\left(\mathbf{S}^{-1}+\mathbf{E}^{T} \mathbf{R}^{-1} \mathbf{E}\right)^{-1} \mathbf{E}^{T} \mathbf{R}^{-1} \delta \mathbf{y} \\
\mathbf{P} & =\left(\mathbf{S}^{-1}+\mathbf{E}^{T} \mathbf{R}^{-1} \mathbf{E}\right)^{-1}
\end{aligned}
$$

where $\mathbf{S}$ is the assumed a priori covariance of the uncertainty in $\delta \mathbf{x}$, and $\mathbf{R}$ is the assumed covariance of $\mathbf{n}_{\mathrm{y}}$, which is the uncertainty in $\mathbf{y}$.

\section{Diffuse internal waves}

Figure 9 shows the observed covariance function for the detided, bandpass filtered diffuse internal-wave displacements; the diagonal gives the variance of these bandpass filtered records. Here the variance grows with depth because of the well-known inverse scaling of displacement variance with buoyancy frequency, $N(z)$ (see Fig. 7, WKB results). The displacements are seen to decorrelate rather rapidly with depth, suggesting a diffuse internal-wave mode spectrum rich in high mode number energy. In Fig. 9(lower panel), the 11 by 11 covariance matrix is "unwrapped" into a 121 element vector and plotted with error bars. It must be noted that due to the symmetry of the covariance function there are only 66 independent values, and only these are used in the spectral estimation. Figure 10 shows the estimated mode spectrum with error bars, and of Fig. 9(lower panel) shows the estimated covariance based on the inversion (details to follow). The fit to the covariance is seen to be quite good, except perhaps at the deepest depths where the observed displacement variance is growing a bit too rapidly for the model to capture. Importantly the estimated spectrum is very nearly of the form $H_{D I W}(j) \propto 1 /\left(j^{2}+j_{*}^{2}\right)$ with $j_{*}^{2}=3$. This spectrum can be compared to the previously mentioned study by Yang and Yoo (1999), who found a form $1 /\left(j^{2}+j_{*}^{2}\right)^{1.75 \pm 0.25}$ with $j_{*}=1$. Although the present value of $j_{*}=\sqrt{3}$ is similar to their value, the roll off of the spectra are very different. As stated previously the differences between the two results are easily attributable to the short $2 \mathrm{~h}$ record and the observable of sound speed (which mixes internal-wave and spice effects) in the study by Yang and Yoo (1999). In contrast to deep water the SW06 diffuse internal-wave field has more of its energy concentrated in the lower modes: The GM spectrum has the same shape as the SW06 spectrum but $j_{*}$ is closer to 3 .

A few words concerning the inverse procedure for the diffuse internal-wave spectrum are necessary. For the initial guess, $\mathbf{x}_{0}$, the mode spectrum has the form $\hat{H}_{D I W}(j)$
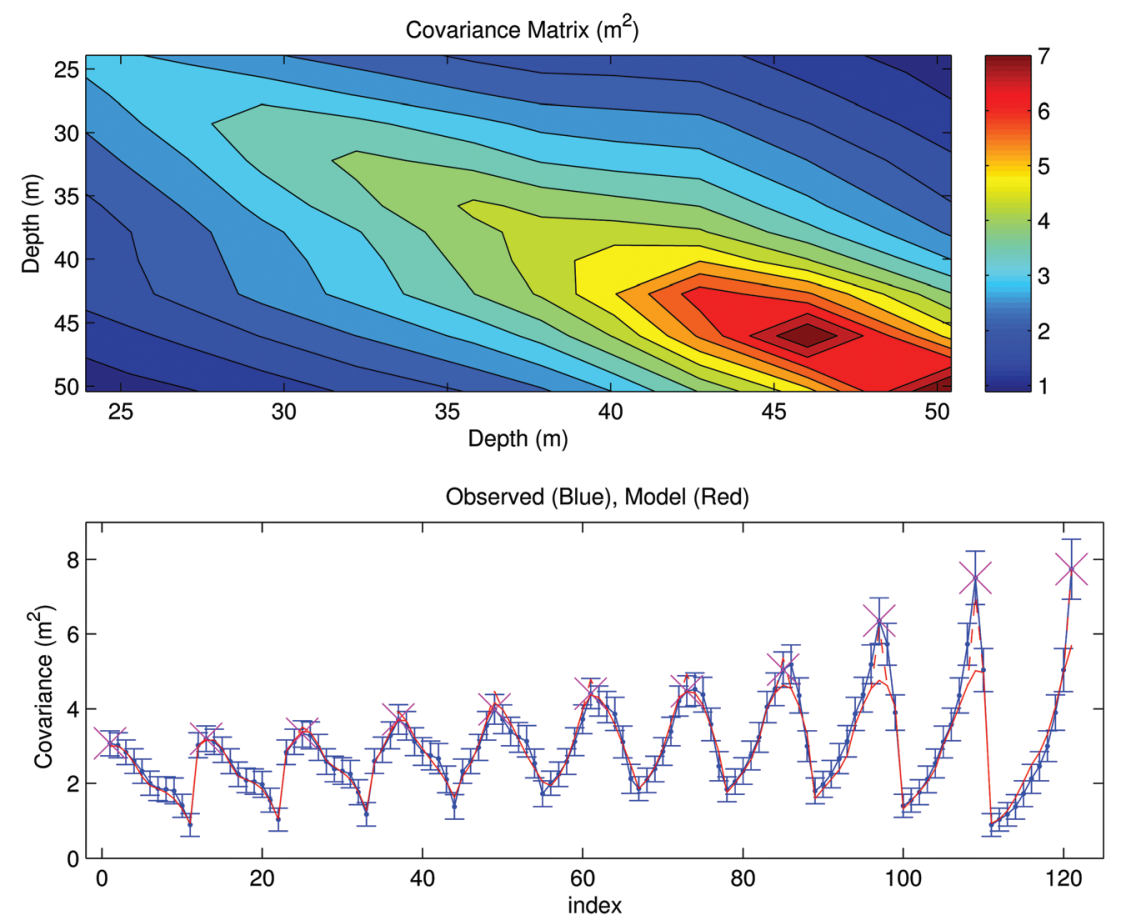

FIG. 9. A contour plot of the covariance matrix $C_{D I W}\left(z_{1}, z_{2}\right)$ for the detided, bandpass filtered internal-wave displacements (upper panel). The lower panel shows the "unwrapped" covariance matrix with error bars, and the diagonal elements (variances) are labeled with magenta crosses. The covariance computed using the estimated mode spectrum $H_{D I W}(j)$ (see Fig. 10) is shown in solid red. The estimated covariance using both the mode spectrum and the noise variance parameters is shown with a red dash. 

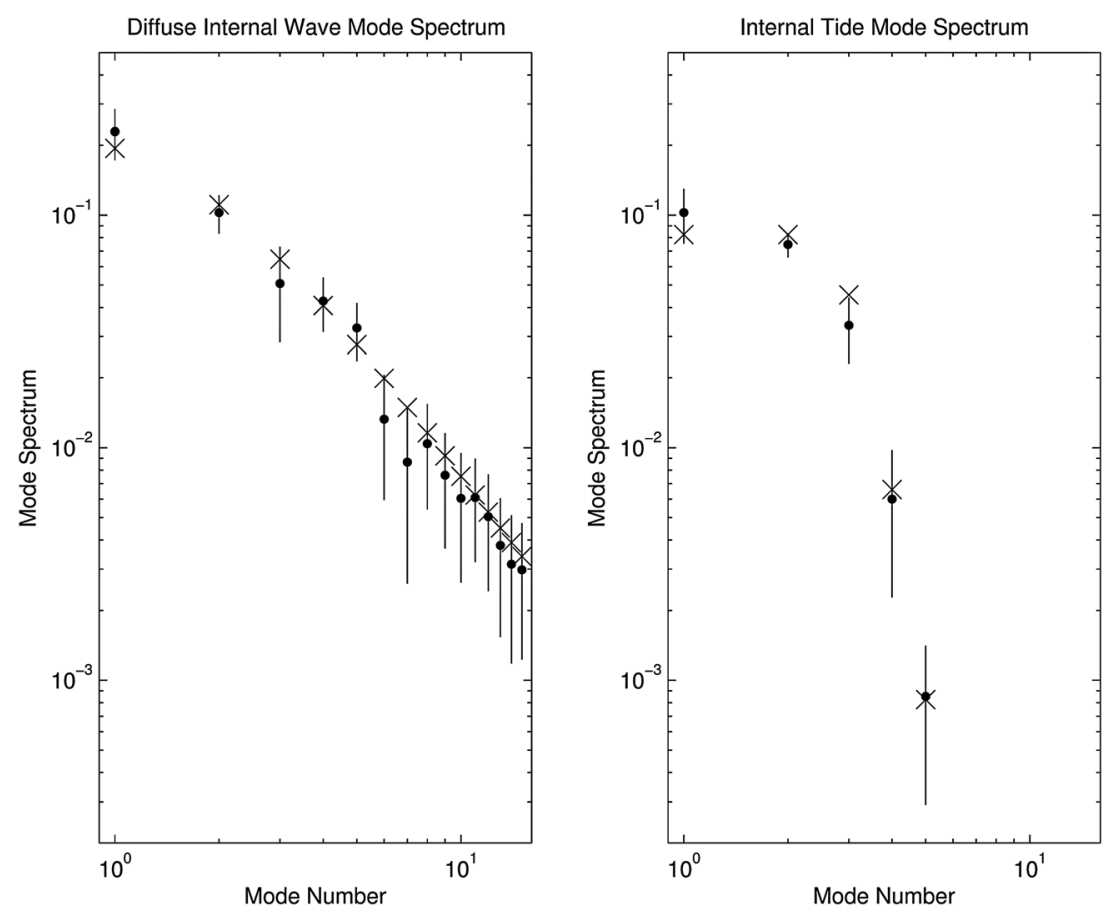

FIG. 10. The estimated mode spectra and uncertainties (dots) for the diffuse internal waves (left) and the internal tide (right). The initial guess spectra are shown with crosses.

$=(0.36)^{2} /\left(j^{2}+2\right)$, and the measurement noise variances, $\epsilon^{2}$, were chosen to increase linearly from $0.01 \mathrm{~m}^{2}$ at the shallowest depth to $1.0 \mathrm{~m}^{2}$ at the deepest depth. These initial choices are consistent with essentially "eye-ball" fits to the observed covariances. Regarding the a priori covariances, namely $\mathbf{S}$ and $\mathbf{R}$, the matrices are assumed to be diagonal with the following form. The mode spectrum part of the matrix $\mathbf{S}$ is given by $0.2 \hat{H}_{D I W}(j) \hat{H}_{D I W}(1)$, which gives a flatter drop off with mode number than the initial guess spectrum. For the measurement noise part of the matrix $\mathbf{S}$, the expected rms deviation of the noise parameter estimates $\epsilon^{2}$ are chosen to be 0.1 $\mathrm{m}^{2}$ at the shallowest depth and increasing linearly to $1.4 \mathrm{~m}^{2}$ at the deepest depth. For the covariance of the errors in the observed covariance matrix, namely $\mathbf{R}$, the values are given by the square of the error bars shown in Fig. 9. It should also be noted that in the inversions a maximum mode number of $J=15$ was chosen.

This maximum mode number corresponds to a minimum vertical wavelength of $\sim 5 \mathrm{~m}$, which is the scale at which one expects the spectrum to cut off due to shear instabilities (Munk, 1981). The matrix E was obtained using internal-wave mode functions $\phi_{j}(z)$ computed from the CTD data presented in Fig. 1. It is found that the estimated spectra are quite insensitive to the details for these specific choices of the a priori information. To be specific, the mode spectrum part of $\mathbf{S}$ was varied between a roll off going as $1 / j^{2}$ and no roll off at all, and noise priors were halved, and set to zero.

\section{Internal tides}

Figure 11 shows the observed covariance function for the internal tide. Again the internal tide variance (the diagonal) is seen to grow with depth consistent with inverse scal- ing with $N(z)$ as discussed previously. The correlation, as expected, is seen to decay much more slowly than that for the diffuse internal waves, suggesting an internal tide spectrum dominated by low modes. Further, the noise variance is seen to be a smaller fraction of the overall variance. The inversion for the internal tide mode spectrum $H_{I T}(j)$ is carried out using only five modes because there is so little energy in the higher modes. The initial guess solution, as before, was chosen based on an "eye-ball" fit to the observed covariance. Here the choice is $\hat{H}_{I T}(j)=0.0167,0.0167$, $0.0092,0.0013$, and $0.0002 \mathrm{~m}^{2}$, and the initial guess measurement noise variance is chosen to increase linearly from $0.0 \mathrm{~m}^{2}$ at the shallowest depth to $0.25 \mathrm{~m}^{2}$ at the deepest point. For the covariance of the deviations from the guess the choice is $\mathbf{S}=0.2 \hat{H}_{I T}(j) \hat{H}_{I T}(1)$ for the mode spectrum part (similar to the DIW case) and $0.0 \mathrm{~m}^{2}$ at the shallow depth increasing linearly to $0.25 \mathrm{~m}^{2}$ for the measurement noise part. As in the diffuse internal-wave case the covariance of the uncertainty in the covariance estimate, $\mathbf{R}$, was determined using the error bars shown in Fig. 11. As in the diffuse internal-wave case the estimated spectrum was found to be insensitive to the specific choices of a priori information.

Figure 10 shows the estimated internal tide mode spectrum with error bars, and the fit to the internal tide covariance is seen in Fig. 11. The fit is satisfactory for the shallower depths but less so for the deeper depths. For the internal tide the incoherent modes approximation is somewhat more dubious and this may account for the less satisfactory fit compared to the diffuse internal-wave spectrum. Here the fit mode spectrum is in the proportion 1.0, 0.74, 0.35, 0.063, and 0.0085 , and the fact that significant energy is seen in the internal tides modes 2 and 3 is surprising, as previous studies have implicated mode one as the strongly dominant mode 

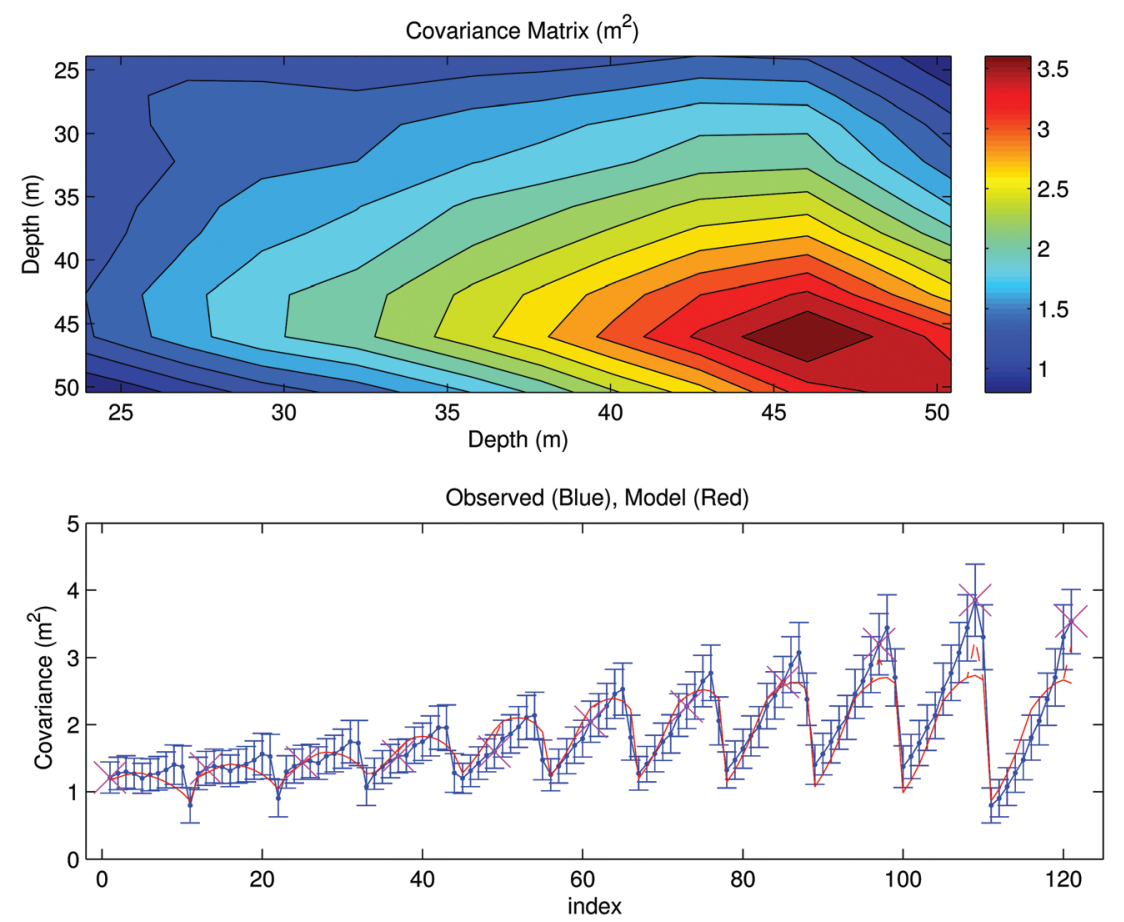

FIG. 11. A contour plot of the covariance matrix $C_{I T}\left(z_{1}, z_{2}\right)$ for the tidal fit internal-wave displacements (upper panel). The lower panel shows the "unwrapped" covariance matrix with error bars, and the diagonal elements (variances) are labeled with magenta crosses. The covariance computed using the estimated mode spectrum $H_{I T}(j)$ (see Fig. 10) is shown in solid red. The estimated covariance using both the mode spectrum and the noise variance parameters is shown with a red dash.

(Apel et al., 1997; Apel et al., 2007). The presence of higher modes is generally associated with internal tide beam propagation near the generation region, or it could be associated with bathymetric coupling of internal tide modes. Future analyses of the extensive SW06 observations and further dynamical modeling may shed some light on this question.

\section{B. Spicy structure}

The covariance analysis discussed in the previous section was also applied to the spicy structure. As potential temperature, salinity, and sound speed are all strongly correlated, any of these properties can be chosen to compute depth covariance; here potential temperature results are presented. Soundspeed results can be easily obtained from potential temperature by multiplying by the square of $3.7 \mathrm{~m} / \mathrm{s} /{ }^{\circ} \mathrm{C}$. It must be noted that in the analysis of the spice variations a band pass filter with cutoff frequencies at the Coriolis frequency $f$ and the cutoff frequency $f_{c}$ was used so as to isolate only the spice fluctuations occurring in the internal-wave frequency band, but excluding the corrupted high frequency fluctuations. The covariance matrix for potential temperature and its errors are shown in Fig. 12. Here, in contrast to the internal-wave displacements, the variance is larger near the surface, which is a reasonable situation as more spice generating mixing is occurring near the surface and the mixed layer (Ferarri and Rudnick, 2000). The spicy structure is seen to decorrelate in depth at about the same rate as the diffuse internal-wave field; this again is likely due to the advection of the spicy structure by the internal-wave horizontal currents. It is also noteworthy that there is very little measurement noise in the spicy covari- ance estimates (i.e., a spike between the zero and first separation) that would be expected if there were strong unresolved nonlinear depth structure. Last, the spice vertical covariance functions estimated here provide critical information that can be used in acoustic fluctuation calculations. However, the acoustic theories also require horizontal correlation function information. Future measurements will be needed to quantify these statistics so that spice induced acoustic effects can be estimated.

\section{SUMMARY}

In this work the SW06 temporal and vertical scales of sound-speed fluctuation caused by internal waves and spicy thermohaline structure have been quantified by tracking the vertical motion and $T / S$ fluctuations of constant density surfaces. The temporal and vertical scales of internal waves and spice are seen to be quite similar presumably due to the horizontal advection of the spicy fine structure by internalwave currents. The vertical distributions of rms sound-speed, however, are quite different for these two processes. Spicy sound-speed structure is seen to dominate the sound-speed variance at all depths considered, suggesting the importance of future measurements of the spatial and temporal distribution of intrusions on the continental slope and shelf. Clearly, increased focus on the study of the effects of spice on shallow-water acoustic propagation will be necessary.

Although the detailed characterization of the nonlinear internal waves is beyond the scope of the present analysis, several important results have been obtained concerning the diffuse internal-wave field and the internal tides. Regarding 

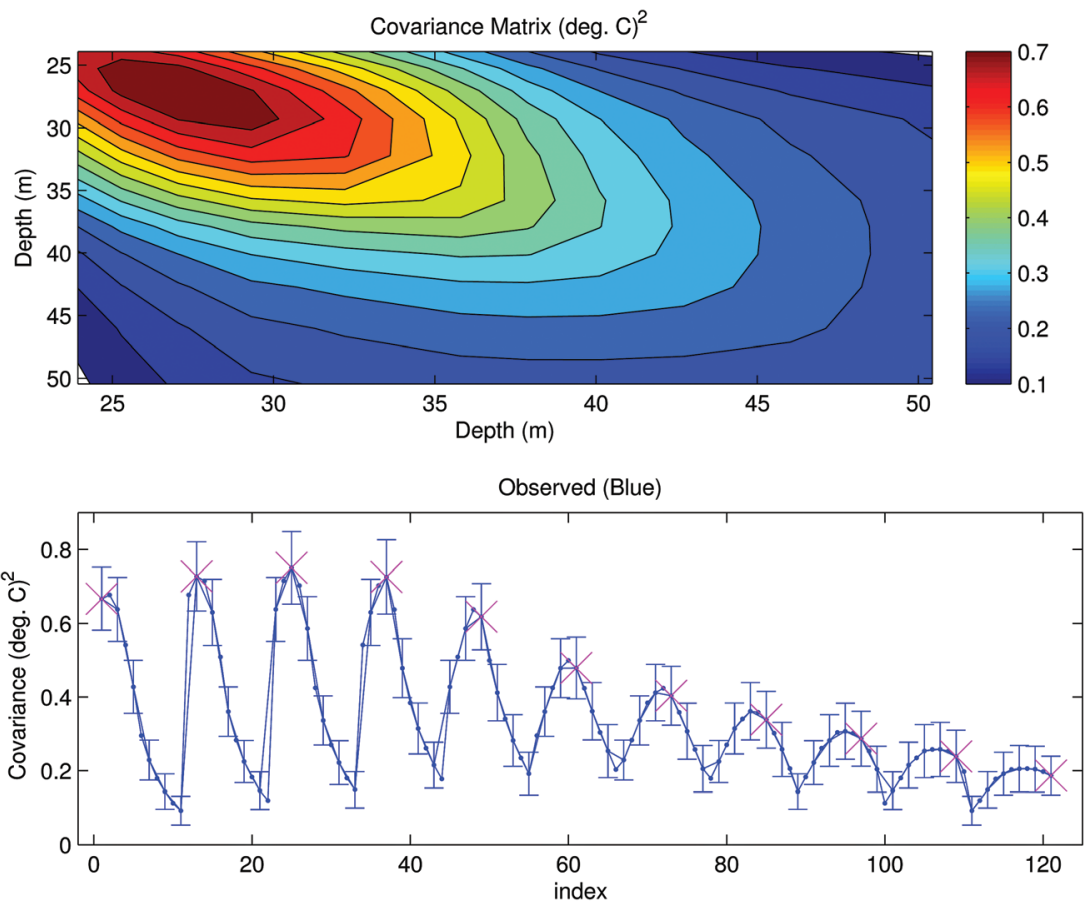

FIG. 12. A contour plot of the covariance matrix $C_{S}\left(z_{1}, z_{2}\right)$ for the spicy temperature fluctuations bandpass filtered in the internal-wave band between $f$ and $f_{c}$ (upper panel). The lower panel shows the "unwrapped" covariance matrix with error bars, and the diagonal elements (variances) are labeled with magenta crosses.

internal tides, this study shows that the semidiurnal tide is not dominated by the first baroclinic mode, and that the sound-speed variance is comparable to that obtained for the diffuse internal-wave field. The presence of higher order internal tide modes suggest there are some beam-like qualities of this field (Duda and Rainville 2008), which have survived from the generation region.

With regard to the diffuse broadband internal-wave field, important acoustical issues motivate their characterization. Theoretical considerations and numerical calculations utilizing results from this study have recently shown that these waves can have important effects on the acoustic observable of mean intensity and intensity variance (Colosi et al., 2012). Further, acoustic-mode phase randomization caused by the diffuse internal waves can have important implications for mode coupling physics caused by nonlinear waves, such that the scattering by these nonlinear waves is much less coherent (Colosi et al., 2012). The analysis of this paper provides some important insights into the frequency and mode number spectra for the SW06 experiment. With regards to the frequency spectrum a reasonable spectral shape is given by $\left(\omega^{2}-f^{2}\right)^{1 / 2} / \omega^{p}$ with $p=-2$, whereas the mode number spectrum is close to the form $1 /\left(j^{2}+3\right)$. Further work will be required to estimate the full 2D spectrum, which may or may not be separable, and most likely will not be horizontally isotropic or homogeneous. As with the spice observations, the question remains as to how representative these results are, and thus future analysis for the spatial and temporal variability of the diffuse fluctuations will be important to correctly characterizing the internal-wave field on the shelf and slope and its changes over seasons. Never the less, the present results will provide important guidance for the interpretation of the SW06 transmission fluctuations.

\section{ACKNOWLEDGMENT}

This work was supported by the Office of Naval Research, and Professor Colosi gratefully acknowledges his additional support from the Naval Postgraduate School's Undersea Warfare Chair that he holds.

\footnotetext{
${ }^{1}$ Thermal models have been developed for various commercially available conductivity sensors to compensate for the response time problem, particularly for temperature, conductivity, depth (CTD) applications (Mensah et al., 2007). Although such a model exists for the Sea-Bird Electronics, Inc. conductivity cell SBE4, mated with a SBE4plus temperature probe and a SBE5 pump, no such model exists for the sensor suite of the pumped SBE37 SMP instruments used in our experiment.

${ }^{2}$ The zero decibar ocean surface pressure reference for potential density is often used in the oceanographic literature. Although the pressure at the surface is clearly atmospheric pressure, not zero pressure, the atmospheric values are so low compared to subsurface values that, for all practical purposes, the zero reference is accurate.

${ }^{3}$ The reference rms displacement value of $\xi_{0}=4.5 \mathrm{~m}$ compared to the deep water value of $7.3 \mathrm{~m}$ means that the SW06 internal-wave field has $40 \%$ of the displacement variance of the deep water field.

Apel, J., M., Badiey, C. S., Chiu, S., Finnette, R., Headrick, J., Kemp, J., Lynch, A., Newhall, M., Orr, B., Pasewark, D., Teilbuerger, A., Turgut, K., von der Heydt, and Wolf, S. (1997). "An overview of the 1995 SWARM shallow water internal wave acoustic scattering experiment," IEEE J. Ocean. Eng. 22, 465-500.

Apel, J., Ostrovsky, L.A., Stepanyants, Y. A., and Lynch, J. (2007). "Internal solitons in the ocean and their effect on underwater sound," J. Acoust. Soc. Am. 121, 695-722.

Beron-Vera, F. J., Brown, M. G., Colosi, J. A., Tomsovic, S., Virovlyansky, A. L., Wolfson, M., and Zaslavsky, G. M. (2003). "Ray dynamics in a long range acoustic propagation experiment,” J. Acoust. Soc. Am. 114, 1226-1242.
} 
Colosi, J. A., Beardsley, R. C., Gawarkiewicz, G., Lynch, J. F., Chiu, C., and Scotti, A. (2001). "Observations of nonlinear internal waves on the outer New England continental shelf during the summer shelfbreak PRIMER study," J. Geophys. Res. Oceans 106, 9587-9601.

Colosi, J. A., and Morozov, A. (2009). "Statistics of normal mode amplitudes in an ocean with random sound speed perturbations: Cross mode coherence and mean intensity," J. Acoust. Soc. Am. 126(3), 1026-1035.

Colosi, J. A., Duda, T. F., and Morozov, A. (2012). "Statistics of lowfrequency normal-mode amplitudes in an ocean with random sound-speed perturbations: Shallow-water environments," J. Acoust. Soc. Am. 131(2), 1749-1761.

Duda, T., Lynch, J., Irish, J., Beardsley, R., Ramp, S., Chiu, C. S., Tang, T., and Yang, Y. (2004). "Internal tide and nonlinear internal wave behavior at the continental slope in the Northern South China Sea," IEEE J. Ocean. Eng. 29, 1105-1130.

Duda, T. F., and Rainville, L. (2008). "Diurnal and semidiurnal internal tide energy flux at a continental slope in the South China Sea," J. Geophys. Res., [Oceans] 113, C03025.

Dzieciuch, M. A., Munk, W. H. and Rudnick, D. (2004). "Propagation of sound through a spicy ocean, the sofar overture," J. Acoust. Soc. Am. 116, $1447-1462$.

Ewart, T. (1980). "A numerical simulation of the effects of oceanic fine structure on acoustic transmission," J. Acoust. Soc. Am. 67, 496-503.

Ferarri, R., and Rudnick, D. L. (2000). "Thermohaline variability in the upper ocean,” J. Geophys. Res. 105, 16857-16883.

Garrett, C., and Munk, W. (1971). "Internal wave spectra in the presence of fine-structure," J. Phys. Oceanogr. 1, 196-202.

Gregg, M. C., and Cox, C. S. (1972). "The vertical microstructure of temperature and salinity,” Deep-Sea Res. 19, 355-376.
Lynch, J. F., Colosi, J., Gawarkeiwicz, G., Duda, T., Pierce, A., Badiey, M., Katsnelson, B., Miller, J., Seigmann, W., Chiu, C. S., and Newhall, A. (2005). "Consideration of finescale oceanography and 3-D acoustic effects for the ESME sound exposure model," IEEE J. Ocean. Eng. 31, $33-48$

Lynch, J., and Tang, D. J. (2008). "Overview of shallow water JASA EL Special Issue Papers," J. Acoust. Soc. Am. 124(3), Pt. 2, EL63-EL65.

Mensah, V., LeMenn, M., and Morel, Y. (2007). "Thermal mass correction for the evaluation of salinity," J. Atmos. Ocean. Technol. 26, 665-672.

Munk, W. (1981). "Internal waves and small scale processes," in The Evolution of Physical Oceanography, edited by B. Warren and C. Wunsch (MIT, Cambridge Press, Cambridge, MA), pp. 264-291.

Munk, W., Worcester, P., and Wunsch, C. (1995). Ocean Acoustic Tomography, Chap. 3, (Cambridge University Press, Cambridge, MA).

Percival, D. B., and Walden, A. T. (1993). it Spectral Analysis for Physical Applications: Multitaper and Conventional Univariate Techniques (Cambridge University Press, Cambridge, MA).

Phillips, O. M. (1971). "On spectra measured in an undulating layered medium," J. Phys. Ocean. 1, 1-4.

Tally, D. L., Pickard, G. L., Emergy, W. J., and Swift, J. H. (2011), Descriptive Physical Oceanography: An Introduction, Chaps. 1 and 8 (Elsevier Academic, New York).

Thompson, D. J. (1982). "Spectrum estimation and harmonic analysis," Proc. IEEE 70, 1055-1096.

Yang, T. C., and Yoo, K. (1999), "Internal wave spectrum in shallow water: Measurement and comparison with the Garrett-Munk model," IEEE J. Ocean. Eng. 24, 333-345.

Young, W. R. (1994). "The subinertial mixed layer approximation,” J. Phys. Ocean. 24, 1812-826. 